\title{
FORMULAE FOR ABSOLUTE MOMENTS
}

\author{
B. M. BROWN
}

(Received 28 October 1969)

Communicated by P. D. Finch

The purpose of this note is to derive an alternative expression to that given in Lemma 1 below (due to Hsu, and to von Bahr) for the absolute moments of a random variable, in terms of the characteristic function.

Let $X$ be a random variable (r.v.) with distribution function (d.f.) $F(x)$ and characteristic function (ch.f.)

$$
\phi(t)=\int_{-\infty}^{\infty} e^{i x t} d F(x) .
$$

The $r^{\text {th }}$ moment of $X$ (or of $F$ ) is

$$
E X^{r}=\mu_{r}=\int_{-\infty}^{\infty} x^{r} d F(x),
$$

and the $r^{\text {th }}$ absolute moment of $X$ (or of $F$ ) is

$$
E|X|^{r}=\beta_{r}=\int_{-\infty}^{\infty}|x|^{r} d F(x) .
$$

When $\beta_{r}<\infty, \phi(t)$ is $r$ times differentiable with

$$
\begin{aligned}
& \phi^{(r)}(0)=i^{r} \mu_{r}, \quad \text { and } \\
& \phi^{(r)}(t)=i^{r} \int_{-\infty}^{\infty} x^{r} e^{i t x} d F(x) ; \quad r=1,2, \cdots
\end{aligned}
$$

(e.g. Lukacs [4], p. 29).

It is well known that the moments $\mu_{r}, r=1,2, \cdots$ can be identified as the coefficients of $(i t)^{r} r$ in a power series expansion of $\phi(t)$ (see Pitman [5], Loeve [3], p. 199, or equations (1), (2) of [1]), thus including absolute moments of even integer order. When $v>0$ is not an even integer, absolute moments $B_{v}$ of order $v$ can be found from the following formula, due to Hsu [2], and von Bahr [6] (see also lemma 1 of [1]).

LEMMA 1. If $v>0$ is not an even integer, and $\beta_{v}<\infty$, then

$$
A_{v} \beta_{v}=\int_{0}^{\infty} \mathscr{R} l\left(\phi(t)-\sum_{j=0}^{m} \frac{(i t)^{j} \mu_{j}}{j !}\right) t^{-(v+1)} d t
$$


where $v=m+\delta$ with $m$ an integer, $0<\delta \leqq 1$, and

$$
A_{v}=-\pi / 2 \Gamma(v+1) \cdot \sin (v \pi / 2) \text {. }
$$

Now assume in addition that $X \geqq 0$ a.e., and let

$$
G_{r}(x)=\int_{0}^{x} u^{r} d F(u)
$$

with $r=0,1, \cdots, m<v \leqq m+1$ and $\beta_{v}<\infty$. Then $\beta_{v}=\mu_{v}$ is the $(v-r)^{\text {th }}$ moment of $G_{r}(x)$ and $i^{-r} \phi^{(r)}(t)$ is the ch.f. of $G_{r}(x)$. Let

$$
\alpha_{m}(t)=\phi(t)-\sum_{j=0}^{m}(i t)^{j} \mu_{j} / j !
$$

According to Theorem 2 of [1], $\mu_{v}=\beta_{v}<\infty$ implies that

$$
\begin{aligned}
\alpha_{m}(t) & =o\left(|t|^{v}\right) \text { for non-integral } v, \\
\mathscr{R} l \alpha_{m}(t) & =o\left(|t|^{v}\right) \text { for odd integers } v, \text { and } \\
\mathscr{I} m \alpha_{m}(t) & =o\left(|t|^{v}\right) \text { for even integers } v, \text { as } t \rightarrow 0 .
\end{aligned}
$$

Applying this result to $G_{r}(x)$ and its ch.f. $i^{-r} \phi^{(r)}(t)$ (noting that $v$ is either non-integral or that $v, v-1, v-2, \cdots$ are odd, even, odd, $\cdots$ integers respectively) gives

LeMma 2. If $v>0$ is not an even integer, $v=m+\delta$ with $m$ an integer and $0<\delta \leqq 1, X \geqq 0$ a.e. and $\beta_{v}=\mu_{v}<\infty$, then

$$
\begin{aligned}
\mathscr{R} l\left(\phi^{(r)}(t)-i^{r} \sum_{j=0}^{m-r}(i t)^{j} \mu_{j+r} / j !\right)=o\left(|t|^{v-r}\right) & \text { as } t \rightarrow 0 ; \\
& \text { for } r=0,1,2, \cdots m .
\end{aligned}
$$

\section{Corollary 1.}

$$
\begin{array}{ll}
\mathscr{R} l\left(\phi^{(r)}(t)-i^{r} \sum_{j=0}^{m-r}(i t)^{j} \mu_{j+r} / j !\right)=o\left(|t|^{\nu-r}\right) & \text { as }|t| \rightarrow \infty, \\
& \text { for } r=0,1,2, \cdots m .
\end{array}
$$

ProOF. Observe that $v>m$ and that $\left|\phi^{(r)}(t)\right| \leqq \mu_{r}<\infty$ for $r \leqq m$.

From lemma 1,

$$
A_{v} \mu_{v}=\lim _{\substack{\varepsilon \rightarrow 0 \\ R \rightarrow \infty}} \int_{\varepsilon}^{R} \mathscr{R} l \alpha_{m}(t) t^{-(v+1)} d t,
$$

which, after integrating by parts $m$ times and invoking Lemma 2 and Corollary 1 for $r=0,1,2, \cdots m-1$ gives

COROLlary 2.

$$
A_{v} \beta_{v}=\lim _{\substack{\varepsilon \rightarrow 0 \\ R \rightarrow \infty}} \frac{\Gamma(1+\delta)}{\Gamma(v+1)} \int_{\varepsilon}^{R} \mathscr{R} l\left(\phi^{(m)}(t)-i^{m} \mu_{m}\right) t^{-(1+\delta)} d t
$$


If $\phi(t)$ is $(m+1)$ times differentiable, then a further integration by parts, and application of Lemma 2 and Corollary 1 for $r=m$, gives

COROLlary 3.

$$
A_{v} \beta_{v}=\lim _{\substack{\varepsilon \rightarrow 0 \\ R \rightarrow \infty}} \frac{\Gamma(\delta)}{\Gamma(v+1)} \int_{\varepsilon}^{R} \mathscr{R} l \phi^{(m+1)}(t) \cdot t^{-\delta} d t
$$

Now drop the assumption that $X \geqq 0$ a.e. Let $X_{+}=\max (0, X), X_{-}=\max$ $(0,-X)$, with $X_{+}, X_{-} \geqq 0$ and $X=X_{+}-X_{-}$. If $\phi_{+}(t), \phi_{-}(t)$ are the ch.fs of $X_{+}$ and $X_{-}$, respectively, then

$$
\begin{aligned}
\phi(t) & =\phi_{+}(t)+\phi_{-}(-t)-1, \\
\mathscr{R} l \phi(t) & =\mathscr{R} l\left(\phi_{+}(t)+\phi_{-}(t)-1\right), \\
\mathscr{R} l \phi^{(r)}(t) & =\mathscr{R} l\left(\phi_{+}^{(r)}(t)+\phi_{-}^{(r)}(t)\right), \quad \text { and } \\
\beta_{v} & =E|X|^{v}=E X_{+}^{v}+E X_{v}^{-} .
\end{aligned}
$$

Therefore, applying Corollaries 2 and 3 to $X_{+}$and $X_{-}$, and adding, gives

THEOREM 1. If $v>0$ is not an even integer, $v=m+\delta$ with $m$ an integer and $0<\delta \leqq 1$, and $\beta_{v}<\infty$, then (1) holds.

If $\phi(t)$ is $(m+1)$ times differentiable, then (2) holds.

We note that (i) $\mu_{0}=1$, (ii) $\phi^{(m+1)}(t)$ might exist for $t \neq 0$ even if $\phi^{(m+1)}(0)$ does not exist, (iii) the integrands in (1), (2) might not be absolutely integrable and thus (iv) contour integration might be needed to evaluate (1) and/or (2).

The possibility of obtaining Theorem 1 arose out of a discussion with Dr. G. K. Eagleson.

\section{References}

[1] B. M. Brown, 'Characteristic functions, moments, and the central limit theorem', Ann. Math. Statist. 41 (1970), 658-664.

[2] P. L. Hsu, 'Absolute moments and characteristic functions', J. Chinese Math. Soc., 1 (1951) 259-280.

[3] M. Loeve, Probability theory, (2nd ed.) (van Nostrand, Princeton, 1960).

[4] E. Lukacs, Characteristic functions (Griffin, London, 1960).

[5] E. J. G. Pitman, 'Some theorems on characteristic functions of probability distributions', Proc. IVth Berk. Symp., (1960), 393-402.

[6] Bengt von Bahr, 'On the convergence of moments in the central limit theorem', Ann. Math. Statist. 36 (1965), 808-818.

La Trobe University 